\title{
Influence of intermediate foil on air-foil bearings performance and exploitation properties \\ (STA162-15)
}

\author{
Jakub Łagodziński, Kacper Miazga
}

\begin{abstract}
Air-foil technology is an interesting alternative for classic rolling or oil bearings in turbomachinery. It ensures low power losses, do not require external lubrication or pressurization and is capable of working with high rotational speeds. Numerous studies brought significant improvement in materials and design solutions for the aerodynamic shaft support system. The paper presents effects of modification performed by adding intermediate foil to typical structure of air-foil bearing. The main aim of the investigation was focused on analyze of its influence on characteristic parameters and exploitation properties of operating bearing, i.e. bearing load capacity and bearing resistance to thermal runaway phenomenon [4]. The thermal runaway is also known as foil bearing thermal instability. The tests confirmed proper behavior of the modified device and brought new knowledge in air-foil technology field.
\end{abstract}

\section{Introduction}

The last decades brought remarkable progress in the field of high speed turbomachinery. New kinds of air cycle devices are designed with consideration of decreasing their dimensions and ability to work with high rotational frequencies. Moreover great attention is paid to limitation of power losses and assurance of reliability.

In order to meet such demands new kinds of rotor support systems had to be developed. One of the proposed solutions is an idea of Oil-Free technology, in which air-foil bearings have great potential.

\subsection{Air-foil Technology}

Air-Foil bearings are self-acting aerodynamic bearings that use ambient gas as their working fluid. The main idea of their design is based on two foils placed between shaft and sleeve: bump and top (see figure 1). As the shaft starts rotating, the ambient gas (e.g. air) is pushed between top foil and journal due to its viscosity that creates thin gas film. After reaching characteristic rotational speed called lift-off speed the air gap is continuous and there is no contact between the shaft and the top foil. In this solution the bump foil is considered to be an elastic support for top foil. 


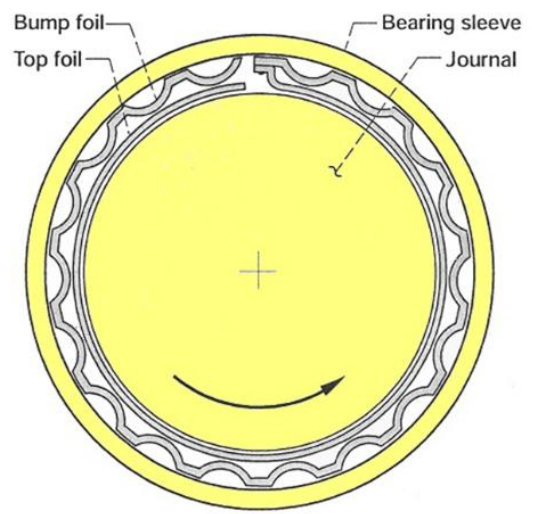

Figure 1. A basic schematic of the air-foil bearing

Air-foil bearings are mainly considered as shaft support system for high rotational speeds cycle machines of low and medium power, usually up to $100 \mathrm{~kW}$. They were firstly applied in Air Cycle Machines and Environmental Control Machines mainly in military aircrafts [1], confirming their high performance abilities. With growing demands and advances in technology the field of use was expanded to many other devices such as blowers, turbojet engines, ORC generators, compressors, turboexpanders etc.

\subsection{Air-foil bearings features and design}

There are many advantages considered with the air-foil bearings. First to be mentioned they are selfacting, aerodynamic supports, therefore, unlike aerostatic bearings, there is no need for external pressurization. It leads to elimination of additional pressure supply systems and simplifying the construction of the machine. Moreover, using ambient gas as working medium air-foil technology is free from oil lubrication, which ensures total cleanliness of working medium. It is significant especially for systems where seals are problematic and any contamination of cycle gas is inadmissible. Furthermore, power losses in machines with air-foil bearings applied are strongly reduced due to their non-contact operation with high rotational speeds.

Despite many advantages, air-foil bearings have significant limitations that have to be considered. First of all there is dry friction phenomenon that occurs during start up and shut down of the machine. Before the thin gas film is formed, the surfaces of the top foil and the shaft have contact with each other. The frictional contact limits the lifetime of the bearing due to surface degradation and leads to destruction of these parts. Solution of this problem lies in application of special coating materials that reduce dry friction and protect foil and shaft from wear. Moreover, such materials should ensure chemical stability, especially where air is not working fluid, good adhesion to the base and solid lubrication possibly. The investigations of coatings brought many different solutions, such 
as ceramics, polymers, metallic materials and composites suitable for wear protection, considering various temperature ranges of operation [2,3]. They can strongly improve the performance of the bearing, ensuring sufficient number of start-stop cycles without undesirable wear.

Another important issue of the air-foil bearings is limited load capacity. With thin self-acting gas film and elastic bump foil as the only support for the journal, the mass of rotating system is limited. If the rotor is too heavy, it can break the continuous gas gap and cause an ineffective operation of the bearing, including damage due to friction.

Under a heavy load, a foil bearing thermal runaway phenomenon may appear. This phenomenon, known also as foil bearing thermal instability, results from too tight fitting in the bearing. This causes positive feedback loop between the frictional torque and journal thermal expansion. The thermal runaway develops very quickly and can cause severe damage to the bearing.

The numerous studies of the air-foil bearing construction brought various concepts for the structure of the device. Main differences lies in the slot-like locks, which are responsible for fixing foils in the sleeve (see figure 2) and elastic support for top foil. The classic bump foil was usually modified by circumferential splits and variable pitch bumps. Another idea was based on substituting bump foil by elastic metal mesh [5]. Numerous different structural variants of air-foil bearings were developed and tested considering multipad, hydresil or multilayer bearings with additional intermediate foil [1].

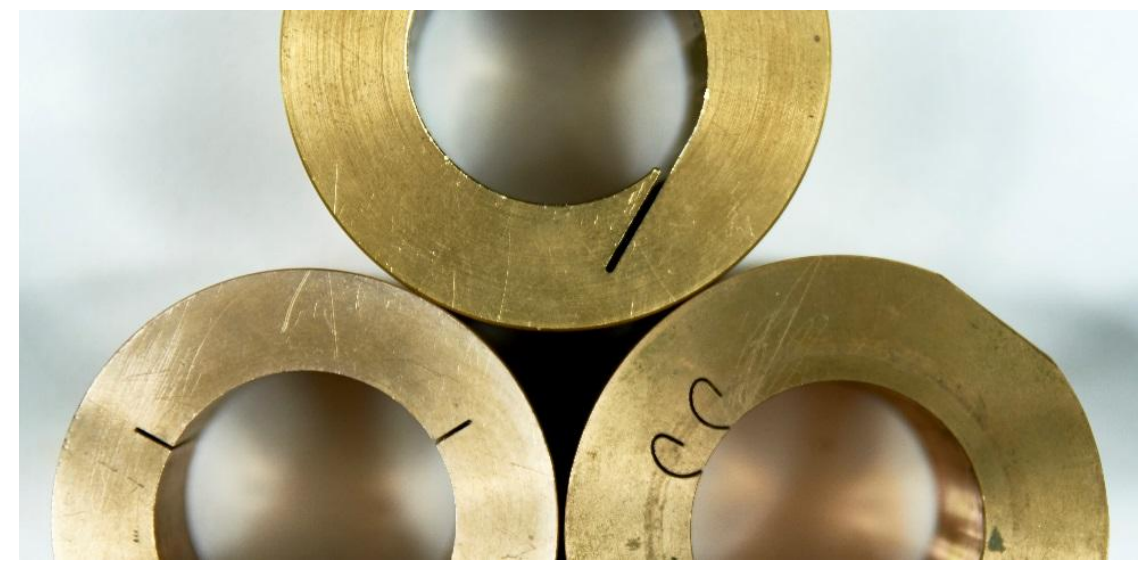

Figure 2. Different types of retaining slots

\section{Intermediate foil in air-foil bearings}

The basic structure of air foil bearing consists of flat top foil and corrugated bump foil which acts as a spring. Most common modifications leading to improvement of the bearing performance were made to bump foils, distinguishing them in three main generations [4][6]. Another interesting adjustment 
was made by introducing intermediate foil into the bearing, which was placed between bump and top foils. This additional element improves damping of the foils structure. Moreover with intermediate foil, the top foil has additional support that decreases a phenomenon of sagging (see figure 3 ). It occurs in collapsing of the top foil between pitches of the bump foil when thin gas film is formed under operation of the bearing [7].
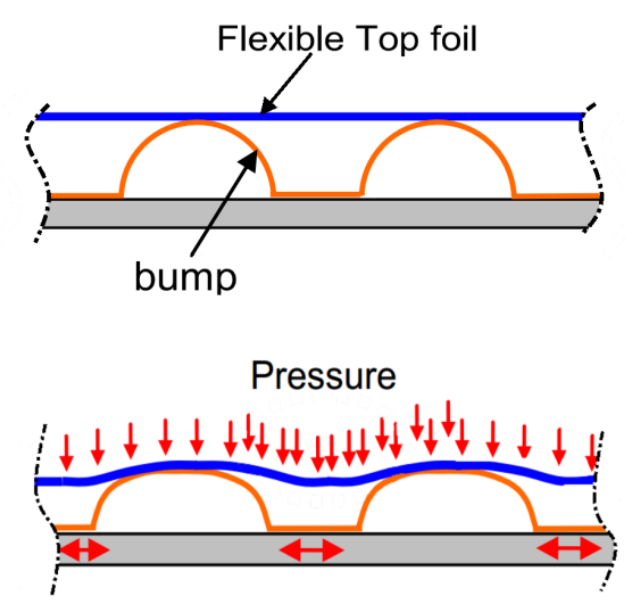

Figure 3. The effect of sagging

During initial tests the intermediate foil occurred to have positive influence on bearing performance. Comparing to basic air-foil bearing construction, the torque at the start-up was reduced. Moreover wear of top foils working in modified bearings was noticeable lower, resulting in increase of the bearing lifetime. These observations brought an idea of tests that could precisely describe influence of intermediate foil in air-foil bearings.

\subsection{The test rig for air foil bearing with intermediate foil.}

The idea of checking the results of intermediate foil implementation was based on comparing a performance of the same bearing with and without this element. The sleeve was prepared to work with three segments of bump foil with circumferential splits, intermediate foil and top foil coated with wear resistant polymer AS20. The shafts coating consisted of chromium oxide ceramic material. The bearing was transformed into classic type by removing intermediate foil and placing three pieces of flat foils with the same thickness as the intermediate between bearings sleeve and bump foil segments. This way it was possible to test two variants of the bearing with the same initial preload, which usually is adjusted by inner diameter of the sleeve [8]. 


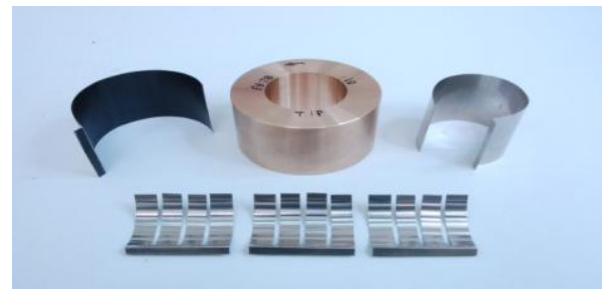

Figure 4. Elements of the air-foil bearing with the intermediate foil.

The test rig was based on electrical spindle controlled by frequency converter. The measured values were rotational speed of the shaft, provided by an optical sensor and bearing torque measured with force gauge attached to its sleeve. The idea of the experiment was to compare a behavior of both variants of bearings working under variable static load. The design of test rig allowed adjustment of static load by suspending a weight on bearings sleeve. In order to eliminate an influence of the weights on force sensor measurement, an externally pressurized aerostatic bearing was placed on the investigated air-foil bearing to provide pendulous attachment of mass.

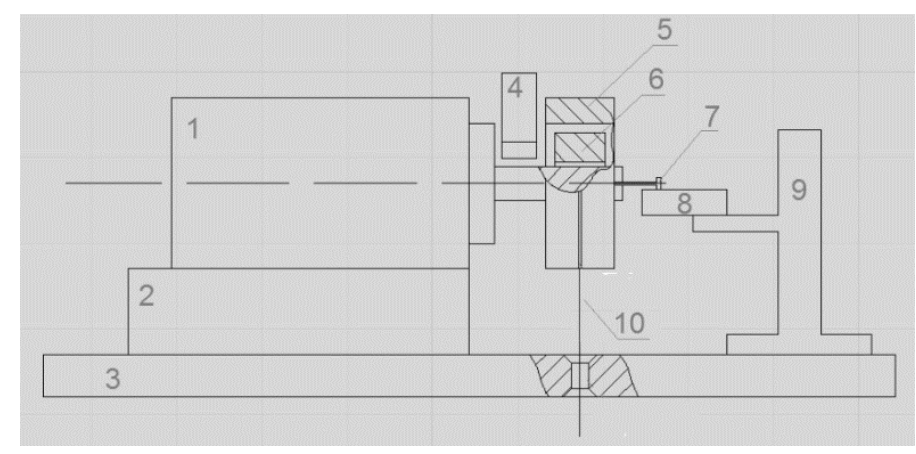

Figure 5. The test rig schematic (1 - electric spindle, 2 - pedestal, 3 - table, 4 - optical sensor, 5 aerostatic bearing, 6 - investigated aerodynamic air-foil bearing, 7 - torque lever, 8 - force sensor, 9 - force sensor rack, 10 - suspension of static load).

\subsection{Load capacity of air-foil bearings.}

The air-foil bearings, as self-acting rotor support system without external pressure supply, are usually used in low and medium power applications, with limited mass of shaft and rotor. The design of the machine needs to consider a specific nature of their performance.

The analysis of air-foil bearings capacity is a strongly complicated issue. Comparing to other types of bearings, the behavior of elastic structure of foils is difficult to describe with equations. Moreover, there has to be considered dependency between thin gas film of working medium and solid structural foils. Another important phenomenon comes from friction between bump and top foils that 
slightly shift their relative position during bearing operation. Considering the complexity of phenomenon that occurs in the air-foil bearings numerical models that can entirely describe their behavior are difficult to create.

Load capacity of the air foil bearing is strongly related to its construction. The experimental data brought a simple method of its estimation. It was shown, that load capacity is linearly related to dimensions of the bearing projected area and surface velocity [9].

Nevertheless, experimental investigations are significant in determining behavior of air-foil bearings in different working conditions. Test performed with variable static load can be used for creating performance map, which is a characteristic based on surface plot that describes power loss in bearing depending on the rotational speed and applied load [10]. With the map, optimal operational points and safety margins can be found.

\section{Experimental results and discussion.}

The experimental tests were performed in two series, beginning with classic type of air-foil bearing, then with the bearing with the intermediate foil. Both devices were loaded with the same static loads forces from $6.3 \mathrm{~N}$ to $113.5 \mathrm{~N}$ (the values considered mass of air-foil and aerostatic bearings sleeves). For each value of load the force on air-foil bearing sleeve was measured and then recalculated to torque and power loss at rotational frequencies from $200 \mathrm{~Hz}$ to $400 \mathrm{~Hz}$ with step of $50 \mathrm{~Hz}$.

\subsection{Results of measurements.}

With collected data the following plots were created, which present operating parameters of both bearings. The characteristics on the Figure 6 present the performance maps of both bearings.
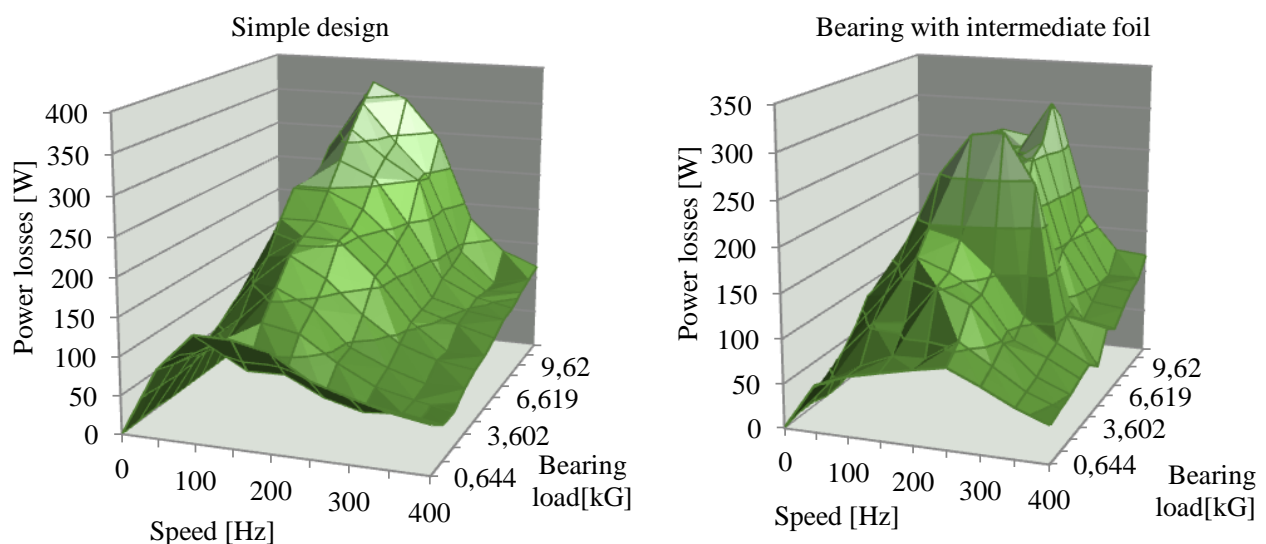

Figure 6. Performance maps. 
In order to get a clear comparison of operation parameters of air-foil bearings with and without the intermediate foil the relation of torque value to suspended static load for specific rotational frequencies of the shaft were plotted.
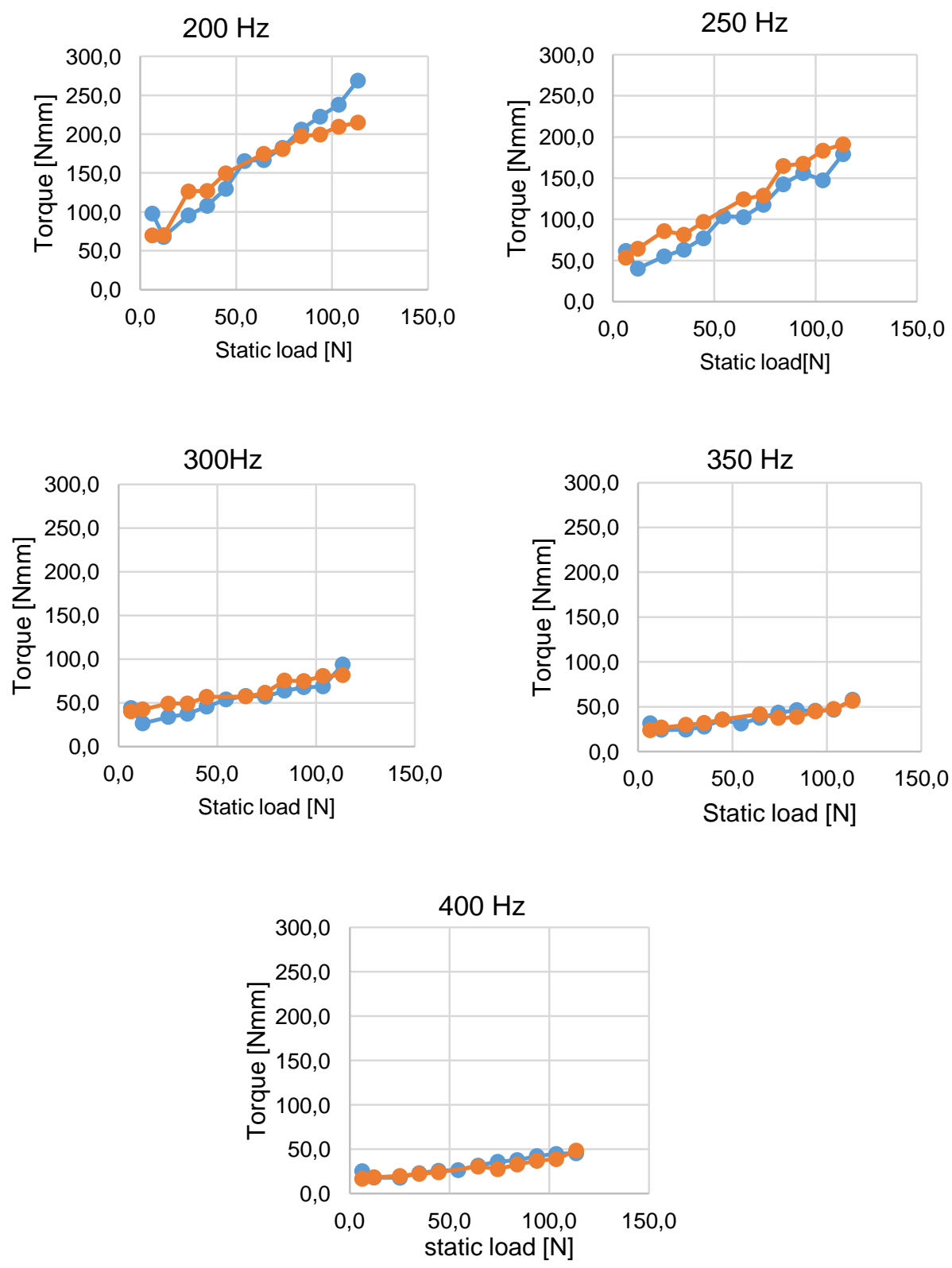

Figure 7. Measured values of torque for specific rotational frequencies of the journal. Note: vertical scales are the same. 
An additional analysis was done to the measured peak values of the torque at the start-up of the bearing for each value of static load, as it is critical moment in operation of the device when dry friction occurs.

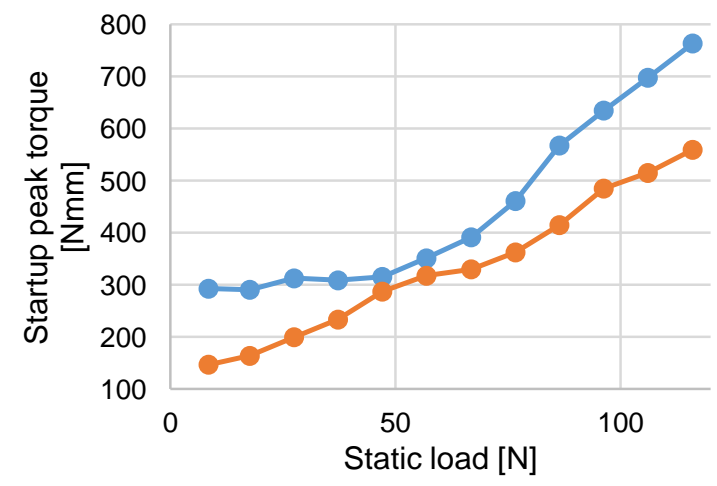

Figure 8. Peak values of measured torque at the star-up of the bearing.

\subsection{Discussion.}

The analysis of the results shows very strong influence of static load on operating parameters of the air-foil bearings. According to former predictions, the measured torque increases with the increase of mass loaded on the bearing sleeve in entire range of rotational speeds of the shaft. In case of implementation of this rotor support system in a machine it would result in growth of power losses with increase of rotating systems mass.

If we take into consideration the power losses dissipated in the bearing (see performance maps in Figure 6), it is clearly visible that applying the intermediate foil can cause a reduction of power losses by $\sim 25 \%$. One can observe, that with simple design, the bearing power losses performance map reaches a peak value of almost 400 Watts, but the bearing with intermediate foil, for the same operating conditions, has a maximum power loss value at only 300 Watts. This means less heat generated during operation, less journal thermal expansion and wider bearing thermal stability margin.

In the Figure 7, were the ranges of all torque axes are the same for all plots, it is found, that static load has bigger influence on measured torque with lower rotational speeds of the shaft. With the increase of rotation frequencies, the lower change of bearings torque is observed with defined increment of static load.

The Figure 7 brings also a comparison of operating parameter of the air-foil bearing with and without intermediate foil. Eventually, there was no significant difference found in performance of both tested variants. The values of measured torque for all rotational speeds and static loads were very 
near. In this case, there cannot be seen any significant influence of the intermediate foil on operation of the air-foil bearing.

The different situation can be observed in plot of measured torque at the star-up of the bearing (see Figure 8). It is a critical moment of the bearings operation where dry friction occurs before formation of the continuous gas film and cause wear of top foils and journals surfaces. In this case the implementation of the intermediate foil causes visible decrease of measured torque. It is translated into reduction of frictional force between the top foil and the journal at the start-up.

The analysis of the results was also based on visual inspection of the top-foils of both bearing variants after tests were performed. It brought an information about level of wear of the coating materials.

a

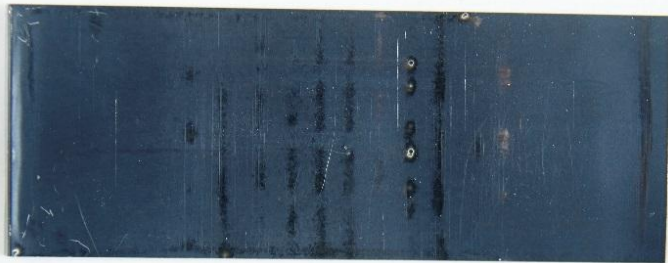

b

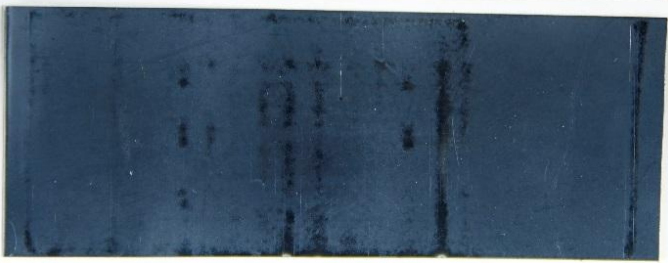

Figure 9. Top foils after test: $\mathrm{a}$ - basic type of the bearing, $\mathrm{b}$ - bearing with the intermediate foil.

The top-foils worked in very similar conditions, being subjected to approximately the same number of start-up and shut-down cycles. The visual inspection showed lower level of wear in AS20 polymer on the top foil which worked in the bearing with implemented intermediate foil. The top-foil from the basic type of the bearing had more degraded surface, even with local complete loss of coating material.

\section{Conclusions.}

The purpose of the experimental tests presented in the paper was to investigate the influence of intermediate foil implemented in the aerodynamic air-foil bearing on operating and exploitation properties of this rotor support system. The evaluation of this variation was based on comparison of the performance of basic and modified bearings working under variable static load. 
The analysis of the test results showed that intermediate foil have a significant influence on power losses in air-foil bearing under operation with continuous gas gap formed. The values of measured torque for both bearings working with the same rotational speeds and suspended static loads varied very slightly. The significant difference was found in measurements of torque at the start-up of the bearings. The application of the intermediate foil caused reduction of measured torque in this critical moment.This indicates decrease in the friction force between the top foil and the journal. The positive effect was also confirmed by visual inspection, which showed lower wear of the top foil that worked in the modified bearing.

The intermediate foil is an interesting modification for aerodynamic air-foil bearings. It improves mutual operation of foils resulting in advances of exploitation properties of the bearing and increases damping by introduction of additional friction between foils and reduces the effect of sagging.

\section{References}

[1] Agrawal, G.L. Foil air/gas bearings technology - an overview. ASME Publication 97-GT-347.

[2] Miazga, K., Tkacz, E., Kozanecki, Z., and Łagodziński, J. Investigation of coating materials for air-foil bearings. Cieplne maszyny przepływowe. Turbomachinery 140, 2011, 149-156.

[3] Hashmat, H., Hryniewicz, P., Walton, J.F., Willis, J.P., Jahanmir, S., and DellaCorte, C. Lowfriction wear-resistant coatings for high-temperature foil bearings. Tribology International 38, 2005, 1059-1075.

[4] DellaCorte, C., Radil, K.C., Bruckner, R.J., and Howard, S.A. Design, Fabrication and performance of open source generation I and II compliant hydrodynamic gas foil bearings. NASA/TM-2007-214691

[5] San Andres, L., and Chirathadam, T., Performance Characteristics of Metal Mesh Foil Bearings: Predictions vs. Measurements. ASME Journal of Engineering for Gas Turbines Power. 135,2013, 1-8.

[6] Nalepa, K., Pietkiewicz, P., and Zywica, G., Developement of the foil bearing technology. Technical Sciences. 12, 2009, 229-240.

[7] San Andres, L., and Kim, T.H., Analysis of gas foil bearings integrating FE top foil models. Tribology International, 42, 2009, 111-120.

[8] Radil, K.C., Howard, S.A., and Dykas, B., The role of radial clearance on the performance of foil air bearing, Tribology Transactions. 45, 2002, 485-490.

[9] DellaCorte, C., and Valco, M.J., Load capacity estimation of foil air journal bearings for oil-free turbomachinery applications. Tribology Transactions. 43, 2000, 795-801.

[10] Radil, K.c., and DellaCorte, C., A three dimensional foil bearing performance map applied to oil free turbomachinery. Tribology Transactions. 53, 2010, 771-778.

Jakub Lagodziński, Ph.D.: Lodz University of Technology, Institute of Turbomachinery. 219/223 Wolczanska St, 90-924 Lodz, Poland. (jakub.lagodzinski@p.lodz.pl). The author gave a presentation of this paper during one of the conference sessions.

Kacper Miazga, M.Sc. (Ph.D. student): Lodz University of Technology, Institute of Turbomachinery. 219/223 Wolczanska St, 90-924 Lodz, Poland. (kacper.miazga@p.lodz.pl) 